\title{
Vasculopatia polipoidal idiopática da coróide: aspectos extremos da evolução da doença em um paciente - Relato de caso
}

\author{
Idiopathic polypoidal choroidal vasculopathy:its extreme aspects in one patient - \\ Case report
}

Ieda Maria Alexandre Barreira ${ }^{1}$ RicardoEvangelista Marrocos de Aragão ${ }^{2}$ Ariosto Bezerra Vale ${ }^{3}$ Joana Gurgel Holanda Filha ${ }^{4}$
Trabalho realizado no Serviço de Oftalmologia do Hospital Walter Cantídeo da Universidade Federal do Ceará - UFC.

Especialista em Retina e Vítreo pela Universidade Federal de São Paulo - UNIFESP - São Paulo (SP).

${ }^{2}$ Doutor em Medicina pela Universidade de Regensburg/ Alemanha. Especialista em Retina e Vítreo pela Universidade de Regensburg/Alemanha. Preceptor de Retina e Vítreo da residência médica de Oftalmologia do Hospital Universitário Walter Cantídeo da Universidade Federal do Ceará - UFC. Professor substituto de Oftalmologia da Faculdade de Medicina da UFC.

Especialista em Catarata e Cirurgia Refrativa pela UNIFESP. Diretor do Hospital de Olhos Dr. René Barreira.

${ }^{4}$ Residente do terceiro ano do Serviço de Oftalmologia do Hospital Universitário Walter Cantídeo da UFC.

Endereço para correspondência: Rua Cel. José Mendonça, 914 - Iguatu (CE) CEP 63500-000

E-mail: ricardomarrocos@yahoo.com

Recebido para publicação em 28.05.2004 Aprovação em 22.11.2004

\begin{tabular}{|l|}
\hline RESUMO \\
\hline O artigo descreve um caso de vasculopatia polipoidal idiopática da \\
coróide (VPIC) e demonstra o papel relevante da indocianinografia no \\
diagnóstico diferencial com outras causas de neovascularização subreti- \\
niana, particularmente degeneração macular relacionada à idade (DMRI), \\
bem como avalia os aspectos extremos na evolução da doença em um \\
único paciente. Paciente de 54 anos, sexo masculino, cor parda, com \\
queixas de baixa de acuidade visual súbita em OD, foi submetido a exame \\
oftalmológico, pelo qual se constatou hemorragia vítrea em OD e, \\
posteriormente, descolamento sero-hemorrágico do epitélio pigmentário \\
da retina em OE. A angiofluoresceinografia e indocianinografia mostra- \\
ram quadro compatível com vasculopatia polipoidal idiopática da coróide. \\
O paciente foi submetido a vitrectomia via pars plana em OD e foi \\
acompanhado por período de 5 meses, evoluindo com perda de visão \\
secundária a descolamento de retina e cicatriz macular disciforme em OD. \\
No olho contralateral, ocorreu involução da vasculopatia com preservação \\
de acuidade visual 20/25. A vasculopatia polipoidal idiopática da coróide \\
é entidade clínica cujas anormalidades do fundo de olho se sobrepõem às \\
manifestações da degeneração macular relacionada à idade exsudativa. A \\
indocianinografia é de vital importância no diagnóstico diferencial entre \\
as duas doenças que tem evolução e prognóstico distintos. Além disso, \\
consideramos este caso muito ilustrativo pelas diferentes nuances com \\
que a doença se manifestou e evoluiu em um único paciente.
\end{tabular}

Descritores: Coróide; Hemorragia da coróide; Fundo de olho; Angiofluoresceinografia; Verde de indocianina; Degeneração macular; Neovascularizaçao coroidal, Relato de caso

\section{INTRODUÇ̃̃̃O}

Vasculopatia polipoidal idiopática da coróide (VPIC), também conhecida como síndrome do sangramento uveal posterior ou síndrome dos descolamentos sero-hemorrágicos múltiplos do epitélio pigmentário da retina $(E P R)^{(1-2)}$, é uma condição incomum que ocorre preferencialmente em indivíduos pigmentados da raça negra e asiática e é mais freqüente em mulheres na faixa etária entre 50 a $60 \operatorname{anos}^{(3)}$.

A VPIC é uma anormalidade da vasculatura coroidal interna consistindo de uma malha vascular dilatada com protuberâncias aneurismática terminais múltiplas em uma configuração polipoidal. Estas lesões polipoidais parecem ser responsáveis por vazamento e sangramento episódico sob o EPR e 
retina sensorial ${ }^{(2)}$. A natureza destas anomalias vasculares foi mais bem evidenciada com o advento da indocianinografia. A VPIC é predominantemente bilateral ${ }^{(3-4)}$ e a ectasia vascular coroidal pode estar localizada perifericamente, na região macular ou mais freqüentemente peripapilar, podendo ser responsável por exsudação aguda, descolamento do EPR serohemorrágico e descolamento da retina ${ }^{(5)}$. Pode haver hemorragia vítrea associada. Este artigo descreve um caso de vasculopatia polipoidal idiopática da coróide com evolução extrema da patologia em um mesmo paciente. $\mathrm{O}$ paciente foi atendido no ambulatório de oftalmologia do Hospital Universitário Walter Cantídio da Universidade Federal do Ceará, onde foi submetido a exame oftalmológico inicial completo com posterior realização de exame angiográfico com indocianina verde e vitrectomia via pars plana (VVPP).

\section{RELATO DE CASO}

Paciente de 54 anos, sexo masculino, pardo, natural e procedente de Fortaleza, procurou nosso serviço com queixa de baixa acuidade visual súbita em olho direito (OD). Era portador de hipertensão arterial sistêmica. Ao exame oftalmológico, apresentava: acuidade visual de percepção luminosa em OD e 20/25 em olho esquerdo (OE), segmento anterior sem anormalidades e pressão intra-ocular de $12 \mathrm{mmHg}$ em ambos os olhos (AO). Ao exame fundoscópico: hemorrragia vítrea em OD e em OE drusas moles em pólo posterior. Ao exame ultra-sonográfico: imagem sugestiva de hemorragia vítrea com retina aplicada. Após três meses, o paciente foi submetido a vitrectomia via pars plana (VVPP), quando foi constatada a presença de cicatriz disciforme em região macular. Paciente evoluiu com descolamento de retina e perda de visão em OD. Após cinco meses de acompanhamento, foi observado descolamento sero-hemorrágico do epitélio pigmentário da retina (EPR) em OE (Figura 1). Exame angiofluoresceinográfico: hiperfluorescência com impregnação tardia correspondendo a drusas e hiperfluorescência tipo "pooling" na área do descolamento seroso com hipofluorescência por bloqueio secundária a hemorragia (Figura 2). A indocianinografia demonstrou alterações vasculares com estruturas polipoidais na margem nasal do descolamento do epitélio pigmentário (Figura 3). Após um período de cinco meses, o paciente mostrou involução da vasculopatia em OE (Figuras 4 e 5), permanecendo com visão de 20/25.

\section{COMENTÁRIOS}

A vasculopatia polipoidal idiopática da coróide é uma entidade clínica que foi descrita pela primeira vez por Yanuzzi em $1982^{(6)}$. A VPIC se caracteriza por alterações vasculares que levam a descolamento seroso ou sero-hemorrágico do EPR e da retina neuro-sensorial, apresentam-se como lesões subretinianas alaranjadas, arredondadas, alongadas ou polipoidais

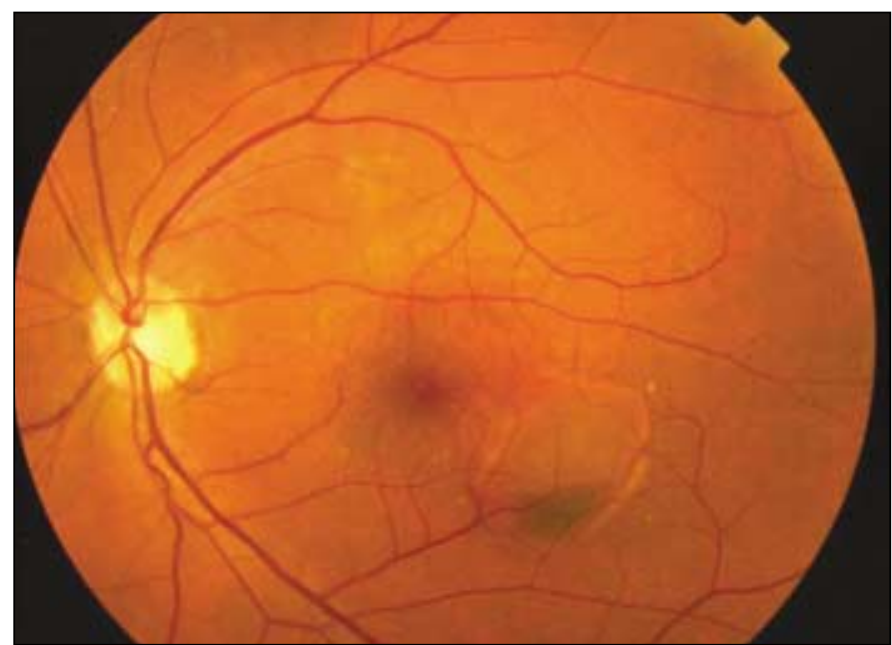

Figura 1 - Descolamento sero-hemorrágico do EPR extra-foveal

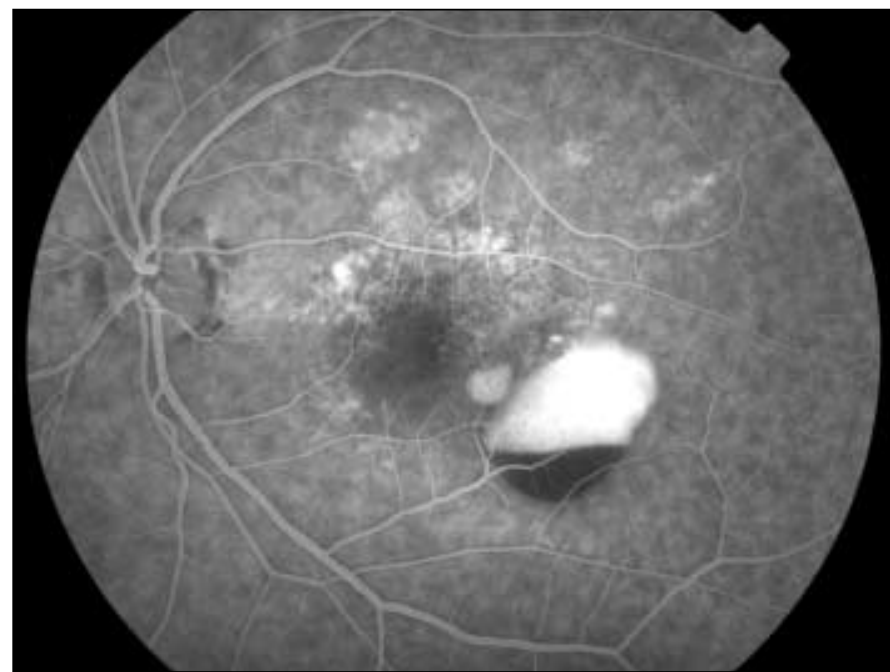

Figura 2 - Hiperfluorescência tipo "pooling" com hipofluorescência por bloqueio na área do descolamento sero-hemorrágico

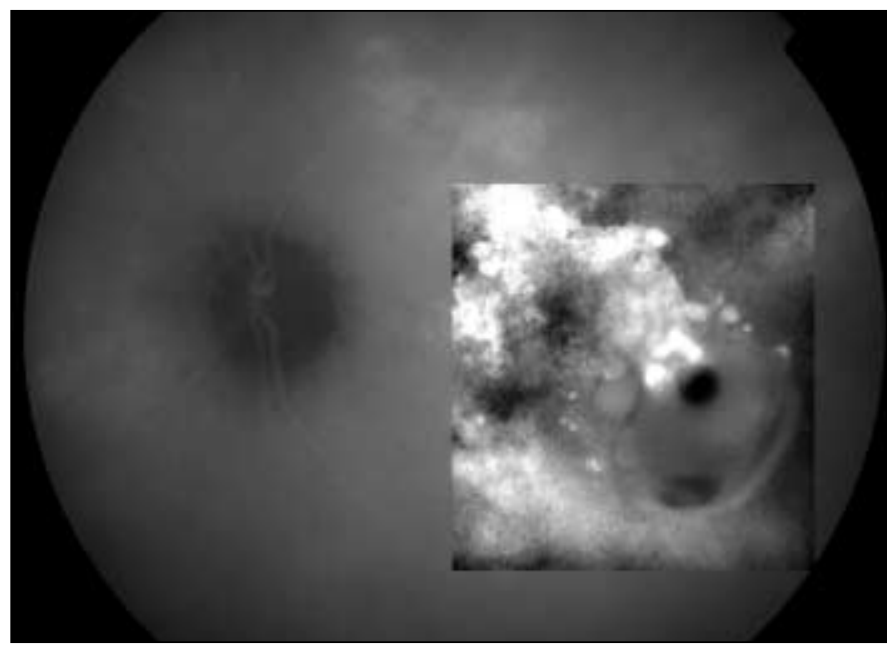

Figura 3 - Estruturas polipoidais da coróide na margem do descolamento sero-hemorrágico 


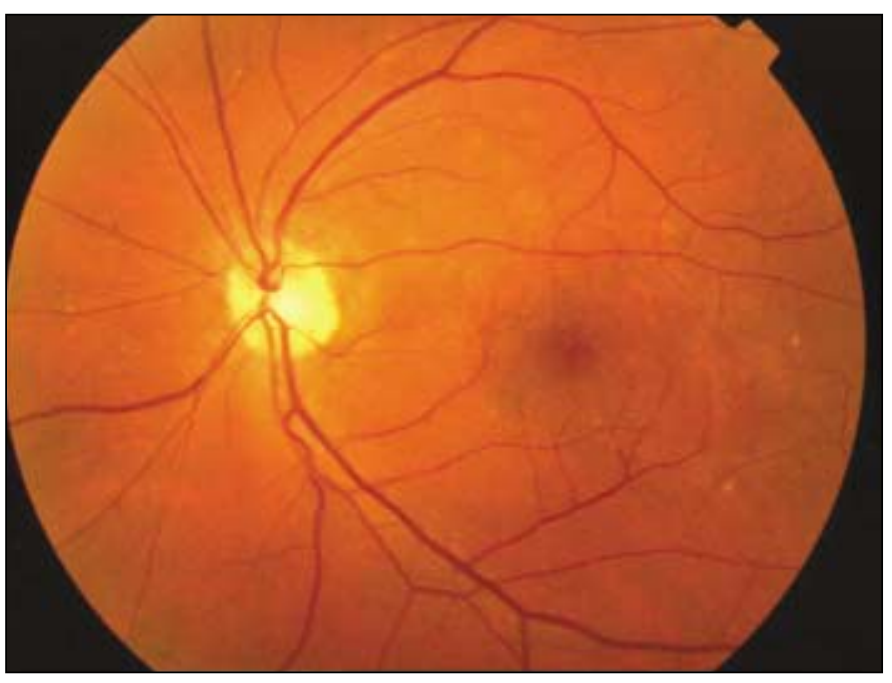

Figura 4 - Regressão do descolamento sero-hemorrágico

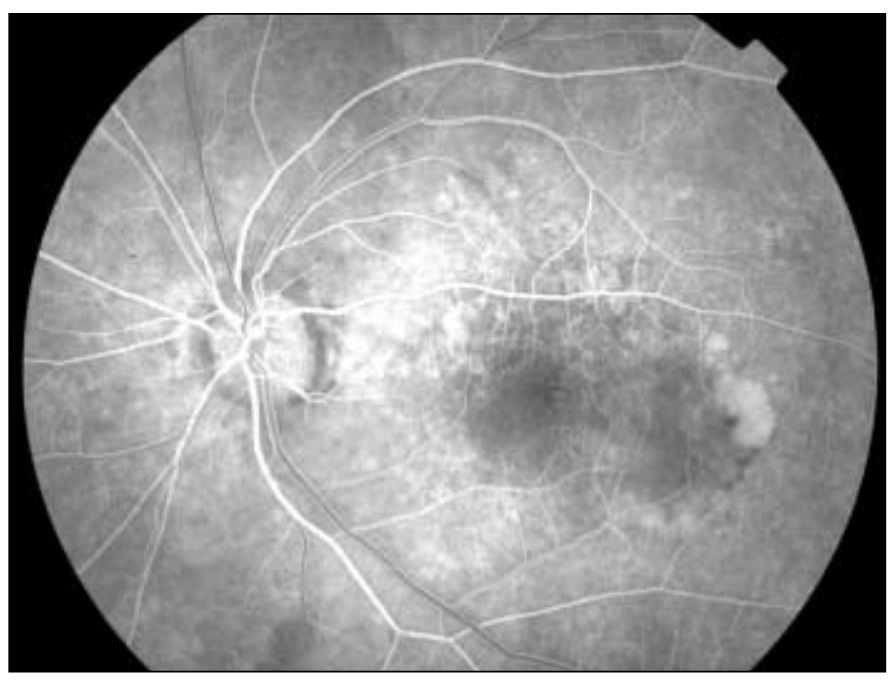

Figura 5 - Hiperfluorescência por defeito em janela secundária a rarefação de epitélio pigmentar da retina após regressão do descolamento sero-hemorrágico

próximo às quais podem ocorrer hemorragias sub-retinianas ${ }^{(7)}$. Dois padrões anatômicos foram descritos: 1) rede de canais vasculares ramificados sob o epitélio pigmentar da retina, geralmente justapapilar em tamanho não padrão da coróide e 2) terminações em nódulos polipoidais vermelho-alaranjados em direção à superfície ${ }^{(8)}$.

A VPIC deve ser diferenciada de outras causas de neovascularização coroidal, incluindo degeneração macular relacionada à idade (DMRI) e coroidopatia serosa central. Yanuzzi e col. observaram que $7,8 \%$ dos pacientes com maculopatia relacionada à idade tinham, na verdade, vasculopatia polipoidal idiopática ${ }^{(9)}$.

Ao considerarmos o diagnóstico de VPIC devemos levar em conta o aspecto epidemiológico (ocorre predominantemente em negros), localização (multifocal), características morfo- lógicas (freqüentemente associada com numerosos descolamentos do EPR) e curso clínico.

A VPIC difere da DMRI pela manutenção da boa acuidade visual. Além disso, a DMRI é mais comum em pacientes brancos caucasianos com distúrbios prévios do EPR macular. Outros diagnósticos diferenciais são as várias causas de neovascularização subretiniana justapapilar como as papilites, estrias angióides e drusas de nervo óptico.

Por se tratar de uma doença primária da coróide, e por estar freqüentemente associada à hemorragia e ao descolamento recorrente do EPR, a indocianinografia define melhor as lesões e é considerado o exame de escolha para o diagnóstico da VPIC já que pode demonstrar as múltiplas áreas de dilatações aneurismáticas e ramificações dos vasos da coróide de médio e grande calibre e também os pontos quentes, importantes para o tratamento com fotocoagulação a laser ${ }^{(6)}$ que, ocasionalmente, é requerido se vazamento serosanguinolento ameaça a fóvea ${ }^{(2)}$.

O manejo ideal dos pacientes com VPIC não está ainda claro. As lesões hemorrágicas podem ter um início fulminante com perda visual severa, mas freqüentemente mostram resolução espontânea. O prognóstico visual parece ser bom, pois muitos pacientes mantêm acuidade visual de 20/80, ou melhor. Hemorragia vítrea ou subretiniana podem, entretanto, causar perda visual severa ${ }^{(10)}$.

O relato deste caso mostra a existência da patologia em nosso meio e confirma o seu prognóstico diverso podendo manter-se assintomático ou levar à perda total de visão, o que ocorreu no caso descrito. O diagnóstico diferencial de VPIC com outras maculopatias exsudativas é de suma importância devido a sua história natural e tratamentos específicos.

\section{ABSTRACT}

To describe a case of idiopathic polypoidal choroidal vasculopathy and the role of indocyanine green angiography findings in the differential diagnosis of exsudative maculopathies, particularly with age-related macular degeneration, and the extreme evolution of idiopathic polypoidal choroidal vasculopathy in one patient. A patient with vitreous hemorrhage was examined and evaluated by fluorescein and indocyanine green angiographies in the right eye and with hemorrhagic detachment of the retinal pigment epithelium in the left eye. The patient was treated by pars plana vitrectomy in the right eye which was followed by retinal detachment and vision loss. In the left eye an involution of the hemorrhagic detachment of the retinal pigment epithelium with preservation of the vision was seen. The idiopathic polypoidal choroidal vasculopathy seems to be a distinct clinical entity that can and should be differentiated from age-related macular degeneration and the fluorescein and indocyanine green angiographies should be performed to evaluate the choroidal vasculature in an attempt to establish a more definitive diagnosis. Particularly in this case the entity had an extreme clinical course in the patient. 
Keywords: Choroid; Choroid hemorrhage; Fundus oculi; Fluorescein angiography; Indocyanine green; Macular degeneration; Choroidal neovascularization, Case report

\section{REFERÊNCIAS}

1. Spaide, RF. ICG Videoangiography of idiopathic polypoidal choroidal vasculopathy. In: Yannuzzi LA, Flower RW, Slakter JS. Indocyanine greeen angiography. St Louis: Mosby; 1997. p.329-39.

2. Kanski JJ. Miscellaneous maculopathies-idiopatic polypoidal choroidal vasculopathy. In: Kanski JJ. Clinical ophthalmology: a systematic approach. Oxford: Butterrworth-Heinemann; 2003. p.434.

3. Yannuzzi LA, Ciardella A, Spaide RF, Rabb M, Freund B, Orlock DA. The expanding clinical spectrum of idiopathic polypoidal choroidal vasculopathy. Arch Ophthalmol. 1997;115(4):478-85.

4. Yannuzzi LA, Freund KB, Goldbaum M, Scassellati-Sforzolini B, Guyer DR, Spaide RF et al. Polypoidal choroidal vasculopathy masquerading as central serous chorioretinopathy. Ophthalmology. 2000;107(4):767-77. Comment in: Ophthalmology. 2001;108(6):1009-10.

5. Quaranta M, Mauget-Faÿsse M, Coscas G. Exudative idiopathic polypoidal choroidal vasculopathy and photodynamic therapy with verteporfin. Am J Ophtalmol. 2002;134(2):277-80.

6. Andrade RE, Castelo Branco A, Costa RA, Uno F, Nakanami M, Guia T, et al. Vasculopatia polipoidal idiopática da coróide. Arq Bras Oftalmol. 2002;65(3):363-6.

7. MacCumber MW, Dastgheib K, Bressler NM, Chan C, Harris M, Fine S et al. Clinicopathologic correlation of the multiple recurrent serosanguineous retinal pigment epithelial detachments syndrome. Retina. 1994;14(2):143-52.

8. Hatanaka M, Tambasco FP, Takahashi WY, Suzuki H, Hirai A. Vasculopatia coroidiana polipóide idiopática - relato de cinco casos. Arq Bras Oftalmol. 2004;67(1):157-60.

9. Yannuzzi LA, Wong DW, Sforzolini BS, Goldbaum M, Tang KC, Spaide $\mathrm{RF}$, et al. Polypoidal choroidal vasculopathy and neovascularized age-related macular degeneration. Arch Ophthalmol. 1999;117(11):1503-10.

10. Moorthy RS, Lyon AT, Rabb MF, Spaide RF, Yannuzzi LA, Jampol LM. Idiopathic polypoidal choroidal vasculopathy of the macula. Ophthalmology. 1998;105(8):1380-5. 